Punjab University Journal of Mathematics (2021),53(12),893-912

https://doi.org/10.52280/pujm.2021.531205

\title{
Solution of nonlinear equations using three point Gaussian quadrature formula and decomposition technique
}

\author{
Gul Sana ${ }^{1 *}$, Muhammad Aslam Noor ${ }^{1}$, Khalida Inayat Noor ${ }^{1}$, \\ ${ }^{1}$ Department of Mathematics, COMSATS University, Islamabad, \\ Park Road Islamabad, Pakistan. \\ E-mail: gulsana123@yahoo.com*,noormaslam@gmail.com \\ ), khalidan@gmail.com
}

Received: 28 April, 2020 / Accepted: 01 Decembre, 2021 / Published online: 24 December, 2021

\begin{abstract}
The problem of solving nonlinear equations (real or complex) is a nontrivial task in many areas of science and engineering. Usually, the analytic methods for such equations are not directly affordable and require an iterative approach for getting an approximate solution. Keeping in view the above facts, we suggest and analyze some new iterative methods for solving nonlinear equation of the form $f(u)=0$ by using the decomposition technique coupled with a system of equations and threepoints Gaussian quadrature formula. We also determine the convergence order of our proposed iterative methods. Some test examples are given to endorse and validate the performance of new methods as compared to previously well-known methods.
\end{abstract}

Key Words: Convergence analysis, Gaussian three point quadrature formula, DaftardarGejji decomposition technique, Nonlinear equation, Iterative methods.

\section{INTRODUCTION}

It is widely known that solving nonlinear equation $f(u)=0$ is one of the key considerable problem in Mathematical sciences particularly in numerical analysis. Many researchers have proposed, analyzed, and modified diversity of numerical methods using different techniques such as: Taylor's series, modified homotopy perturbation method, decomposition method, variational iterative method, and quadrature formulas for numerous enhancements. A vast literature is available that highlight different methods for the solution of nonlinear equations for example, see [1, 5, 7, 8, 10-16, 19-24, 29, 34, 35] and references therein. Abbasbandy [1] has applied ADM (Adomian decomposition method) to present some new modifications in the Newton-Raphson method. Later on, Chun [7] has constructed a sequence of multistep higher-order iterative methods by using the same technique. Implementation of ADM requires the involvement of higher-order polynomial and 
its derivative that is itself a problem. To overcome this weakness of ADM several new techniques have been suggested and analyzed. Another simple decomposition technique was introduced by Daftardar-Gejji and Jafari [9] that does not require higher-order derivative evaluation. The purpose of this technique was to solve a variety of diverse problems and plays a central role in suggesting iterative methods for solving nonlinear equations. Noor et al. $[25,26]$ have used this technique and derived various order families of convergent iterative methods by writing the nonlinear function in a new series representation with the help of quadrature formula and the fundamental theorem of calculus. Saqib and Iqbal [30] have constructed the sequence of multistep iterative methods using this technique and derived a comparative study of numerical results. Alharbi et al. [2] have introduced some new and efficient iterative methods and implemented a decomposition technique along with an auxiliary function. Quadrature rules are another effective and veritable tools that are employed to develop effective iterative methods for getting approximate, converging solutions of the nonlinear equations. Quadrature-based iterative methods are developed by approximating the indefinite integral representation of Newton's method to Taylor's expansion of vector function and employing quadrature formulas. This domain is adressed by considering classical quadrature formulas see [17,34]. Furthermore, the connection has been re-established by Noor [28] and derived the quadrature-based fifth-order convergent method by using the specially derived Gaussian quadrature formula for solving non-linear equations. By implementing the decomposition technique [9], Ogbereyivwe and Muka [32] have developed quadrature-based families of iterative methods for solving system of nonlinear equations subject to singular jacobian. Ali et al. [3,4] have generalized and developed a family of iterative methods. They testified the performance of these methods by numerical and graphical analysis of some nonlinear equations. Based on the Gauss quadrature rule Srivastava et al. [31] have investigated sixth-order three-step Newtons' method for solving a system of nonlinear equations. These methods are also helpful to evaluate nonlinear boundary-value problems and integral equations in fewer iterations with higher accuracy.

Motivated by the research going on in this direction. In this article, we first develop a class of quadrature-based multistep iterative methods by applying decomposition technique and fundamental law of calculus along with the three-point Gaussian quadrature formula [6]. These quadrature-based methods are of implict type, in which we use Newton's method as predictor and iterative function generated from quadrature rule as corrector; see Section 2. Section 3 deals with the convergence analysis and calculations of error equations of these algorithms. Some numerical real-world examples of nonlinear equations are considered in Section 4 to check the efficiency of the predictor-corrector type schemes. Moreover, a comparative analysis of these methods with some other methods is presented to indicate an efficient refinement $\&$ alternative of already developed convergent methods of the same order. Finally, as a part of our concluding remarks, we discuss the accumulated facts of our findings.

\section{NEW CLASS OF FAMILY OF ITERATIVE SCHEMES}

Consider the nonlinear equation

$$
f(u)=0, \quad u \in \mathbb{R} .
$$


Where $\beta$ is an initial guess suffitiently close to $\alpha$ which is simple root of equation (2. 1 ). Now, utilizing the technique of Noor et al. [25,26] approximate the function $f(u)$ using fundamental theorem of calculus and quadrature formula:

$f(u)=f(\beta)+\frac{u-\beta}{18}\left\{5 f^{\prime} \frac{u+\beta}{2}-\frac{u-\beta}{2} \sqrt{\frac{3}{5}}+8 f^{\prime} \frac{u+\beta}{2}+5 f^{\prime} \frac{u+\beta}{2}+\frac{u-\beta}{2} \sqrt{\frac{3}{5}}\right\}$.

Applying the technique of He [18] and writing the nonlinear equation as an equivalent coupled system of equations:

$$
\begin{aligned}
& f(\beta)+\frac{u-\beta}{18}\left\{5 f^{\prime} \frac{u+\beta}{2}-\frac{u-\beta}{2} \sqrt{\frac{3}{5}}+8 f^{\prime} \frac{u+\beta}{2}+5 f^{\prime} \frac{u+\beta}{2}+\frac{u-\beta}{2} \sqrt{\frac{3}{5}}\right\}+g(u), \\
& g(u)=f(u)-f(\beta)-\frac{u-\beta}{18}\left\{5 f^{\prime} \frac{u+\beta}{2}-\frac{u-\beta}{2} \sqrt{\frac{3}{5}}+8 f^{\prime} \frac{u+\beta}{2}+5 f^{\prime} \frac{u+\beta}{2}+\frac{u-\beta}{2} \sqrt{\frac{3}{5}}\right\} .
\end{aligned}
$$

It can be rephrased as

$$
\begin{aligned}
& u=\beta-\frac{18 f(\beta)+g(u)}{\left\{5 f^{\prime} \frac{u+\beta}{2}-\frac{u-\beta}{2} \sqrt{\frac{3}{5}}+8 f^{\prime} \frac{u+\beta}{2}+5 f^{\prime} \frac{u+\beta}{2}+\frac{u-\beta}{2} \sqrt{\frac{3}{5}}\right\}}, \\
& u=c+\mathcal{N}(u) .
\end{aligned}
$$

where

$$
\begin{aligned}
c & =\beta, \\
\text { and } \mathcal{N}(u) & =-\frac{18 f(\beta)+g(u)}{\left\{5 f^{\prime} \frac{u+\beta}{2}-\frac{u-\beta}{2} \sqrt{\frac{3}{5}}+8 f^{\prime} \frac{u+\beta}{2}+5 f^{\prime} \frac{u+\beta}{2}+\frac{u-\beta}{2} \sqrt{\frac{3}{5}}\right\}} .
\end{aligned}
$$

It is clear that $\mathcal{N}(u)$ is a nonlinear operator. Now we construct sequence of higher order iterative methods by employing decomposition technique initiated by Daftardar-Gejji and Jafari [9]. With the support of this technique solution of Eq. (2. 1 ) can be represented as in terms of the infinite series:

$$
u=\sum_{r=0}^{\infty} u_{r}
$$

The nonlinear operator can be decomposed as:

$$
\left.\mathcal{N}(u)=\mathcal{N}\left(u_{0}\right)+\sum_{r=1}^{\infty}\left\{\mathcal{N}\left(\sum_{q=0}^{r} u_{q}\right)-\mathcal{N}\left(\sum_{q=0}^{r-1} u_{q}\right)\right)\right\} .
$$

Thus from equation (2. 6$),(2.9),(2.10)$, we have

$$
\sum_{r=0}^{\infty} u_{r}=c+\mathcal{N}\left(u_{0}\right)+\sum_{r=1}^{\infty}\left\{\mathcal{N}\left(\sum_{q=0}^{r} u_{q}\right)-\mathcal{N}\left(\sum_{q=0}^{r-1} u_{q}\right)\right\}
$$


which generates the following iterative scheme:

$$
\left\{\begin{aligned}
u_{0} & =c \\
u_{1} & =\mathcal{N}\left(u_{0}\right), \\
u_{2} & =\mathcal{N}\left(u_{0}+u_{1}\right)-\mathcal{N}\left(u_{0}\right), \\
& \vdots \\
u_{m+1} & =\mathcal{N}\left(\sum_{q=0}^{m} u_{q}\right)-\mathcal{N}\left(\sum_{q=0}^{m-1} u_{q}\right), \quad m=1,2, \ldots
\end{aligned}\right.
$$

It follows that

$$
u_{1}+u_{2}+\cdots+u_{m+1}=\mathcal{N}\left(u_{0}+u_{1}+u_{2}+\cdots+u_{m}\right), \quad m=1,2, \ldots
$$

and

$$
u=c+\sum_{r=1}^{\infty} u_{r}
$$

Notable approximation of $u$ is conveyed as:

$$
\begin{aligned}
\lim _{m \rightarrow \infty} U_{m} & =u, \\
\text { and } \quad U_{m} & =u_{0}+u_{1}+\cdots u_{m} .
\end{aligned}
$$

For $m=\mathbf{0}$, we have

$$
\mathbf{u} \approx \mathbf{U}_{\mathbf{0}}=\mathbf{u}_{\mathbf{0}}=\mathbf{c}=\beta
$$

It follows from (2. 4$)$ and (2. 7 ), we get

$$
g\left(u_{0}\right)=0 .
$$

Considering (2.8), (2.12) and (2. 17 ), we obtain:

$$
\begin{aligned}
u_{1}=\mathcal{N}\left(u_{0}\right) & =-\frac{18\left(f(\beta)+g\left(u_{0}\right)\right)}{5 f^{\prime}\left(\frac{u_{0}+\beta}{2}-\frac{u_{0}-\beta}{2} \sqrt{\frac{3}{5}}\right)+8 f^{\prime}\left(\frac{u_{0}+\beta}{2}\right)+5 f^{\prime}\left(\frac{u_{0}+\beta}{2}+\frac{u_{0}-\beta}{2} \sqrt{\frac{3}{5}}\right)}, \\
& =-\frac{18(f(\beta))}{5 f^{\prime}\left(\frac{u_{0}+\beta}{2}-\frac{u_{0}-\beta}{2} \sqrt{\frac{3}{5}}\right)+8 f^{\prime}\left(\frac{u_{0}+\beta}{2}\right)+5 f^{\prime}\left(\frac{u_{0}+\beta}{2}+\frac{u_{0}-\beta}{2} \sqrt{\frac{3}{5}}\right)} .
\end{aligned}
$$

For $m=1$, we have

$$
\begin{aligned}
& \mathbf{u} \approx \mathbf{U}_{\mathbf{1}}=\mathbf{u}_{\mathbf{0}}+\mathbf{u}_{\mathbf{1}}=\mathbf{u}_{\mathbf{0}}+\mathcal{N}\left(\mathbf{u}_{\mathbf{0}}\right) \\
& u=\beta-\frac{18 f(\beta)}{5 f^{\prime}\left(\frac{u+\beta}{2}-\frac{u-\beta}{2} \sqrt{\frac{3}{5}}\right)+8 f^{\prime}\left(\frac{u+\beta}{2}\right)+5 f^{\prime}\left(\frac{u+\beta}{2}+\frac{u-\beta}{2} \sqrt{\frac{3}{5}}\right)}, \\
& =u_{0}-\frac{f\left(u_{0}\right)}{f^{\prime}\left(u_{0}\right)} .
\end{aligned}
$$

This formulation suggests the following algorithm: 
Algorithm 2(a): For a given initial guess $u_{0}$, approximate solution $u_{n+1}$ is determined by the following iterative scheme:

$$
u_{n+1}=u_{n}-\frac{f\left(u_{n}\right)}{f^{\prime}\left(u_{n}\right)}, \quad f^{\prime}\left(u_{n}\right) \neq 1, \quad \mathrm{n}=0,1,2, \ldots
$$

This is famous Newton's method which have 2nd order convergence [27].

It is noted that

$$
u_{0}+u_{1}-\beta=-\frac{f(\beta)}{f^{\prime}(\beta)},
$$

From (2. 4$),(2.8)$ and (2. 21$)$, we have

$$
\begin{aligned}
& g u_{0}+u_{1}=f\left(u_{0}+u_{1}\right)-f(\beta)-\frac{f(\beta)}{18 f^{\prime}(\beta)}\left\{5 f^{\prime} \frac{u_{0}+u_{1}+\beta}{2}-\frac{u_{0}+u_{1}-\beta}{2} \sqrt{\frac{3}{5}}\right. \\
& \left.\quad+8 f^{\prime} \frac{u_{0}+u_{1}+\beta}{2}+5 f^{\prime} \frac{u_{0}+u_{1}+\beta}{2}+\frac{u_{0}+u_{1}-\beta}{2} \sqrt{\frac{3}{5}}\right\}, \\
& \begin{array}{l}
u_{1}+u_{2}=\mathcal{N}\left(u_{0}+u_{1}\right)=-\frac{f(\beta)}{f^{\prime}(\beta)} \\
-\frac{18 f\left(u_{0}+u_{1}\right)}{5 f^{\prime} \frac{u_{0}+u_{1}+\beta}{2}-\frac{u_{0}+u_{1}-\beta}{2} \sqrt{\frac{3}{5}}+8 f^{\prime} \frac{u_{0}+u_{1}+\beta}{2}+5 f^{\prime} \frac{u_{0}+u_{1}+\beta}{2}+\frac{u_{0}+u_{1}-\beta}{2} \sqrt{\frac{3}{5}}} .
\end{array}
\end{aligned}
$$

For $m=2$, we have

$$
\mathbf{u} \approx \mathbf{U}_{2}=\mathbf{u}_{0}+\mathbf{u}_{1}+\mathbf{u}_{2}=\mathbf{c}+\mathcal{N}\left(\mathbf{u}_{0}+\mathbf{u}_{1}\right)
$$

$$
\begin{aligned}
& =\beta-\frac{f(\beta)}{f^{\prime}(\beta)} \\
& -\frac{18 f\left(u_{0}+u_{1}\right)}{5 f^{\prime} \quad \frac{u_{0}+u_{1}+\beta}{2}-\frac{u_{0}+u_{1}-\beta}{2} \sqrt{\frac{3}{5}}+8 f^{\prime} \frac{u_{0}+u_{1}+\beta}{2}+5 f^{\prime} \frac{u_{0}+u_{1}+\beta}{2}+\frac{u_{0}+u_{1}-\beta}{2} \sqrt{\frac{3}{5}}}
\end{aligned}
$$

This formulation yields the following two step method for solving nonlinear equation:

Algorithm 2(b): For a given $x_{0}$, approximate solution $x_{n+1}$ is computed by the following iterative scheme:

$$
\begin{gathered}
y_{n}=u_{n}-\frac{f\left(u_{n}\right)}{f^{\prime}\left(u_{n}\right)}, \\
18 f\left(y_{n}\right) \\
u_{n+1}=y_{n}-\frac{1}{5 f^{\prime} \frac{y_{n}+u_{n}}{2}-\frac{y_{n}-u_{n}}{2} \sqrt{\frac{3}{5}}+8 f^{\prime} \frac{y_{n}+u_{n}}{2}+5 f^{\prime} \frac{y_{n}+u_{n}}{2}+\frac{y_{n}-u_{n}}{2} \sqrt{\frac{3}{5}}} \\
\mathrm{n}=0,1,2, \ldots . .
\end{gathered}
$$

It is noted that

$$
\begin{aligned}
& u_{0}+u_{1}+u_{2}=\beta-\frac{f(\beta)}{f^{\prime}(\beta)} \\
& -\frac{18 f\left(u_{0}+u_{1}\right)}{5 f^{\prime} \quad \frac{u_{0}+u_{1}+\beta}{2}-\frac{u_{0}+u_{1}-\beta}{2} \sqrt{\frac{3}{5}}+8 f^{\prime} \frac{u_{0}+u_{1}+\beta}{2}+5 f^{\prime} \frac{u_{0}+u_{1}+\beta}{2}+\frac{u_{0}+u_{1}-\beta}{2} \sqrt{\frac{3}{5}}},
\end{aligned}
$$




$$
\begin{gathered}
g u_{0}+u_{1}+u_{2}=f\left(u_{0}+u_{1}+u_{2}\right)-f(\beta)-\frac{f(\beta)}{18 f^{\prime}(\beta)}\left\{5 f^{\prime} \frac{u_{0}+u_{1}+u_{2}+\beta}{2}-\frac{u_{0}+u_{1}+u_{2}-\beta}{2} \sqrt{\frac{3}{5}}\right. \\
\left.+8 f^{\prime} \frac{u_{0}+u_{1}+u_{2}+\beta}{2}+5 f^{\prime} \frac{u_{0}+u_{1}+u_{2}+\beta}{2}+\frac{u_{0}+u_{1}+u_{2}-\beta}{2} \sqrt{\frac{3}{5}}\right\}, \\
u_{1}+u_{2}+u_{3}=N\left(u_{0}+u_{1}+u_{2}\right)=\beta-\frac{f(\beta)}{f^{\prime}(\beta)} \\
-\frac{u_{0}+u_{1}+\beta}{2}-\frac{u_{0}+u_{1}-\beta}{2} \sqrt{\frac{3}{5}}+8 f^{\prime} \frac{u_{0}+u_{1}+\beta}{2}+5 f^{\prime} \frac{u_{0}+u_{1}+\beta}{2}+\frac{u_{0}+u_{1}-\beta}{2} \sqrt{\frac{3}{5}} \\
-\frac{18 f\left(u_{0}+u_{1}+u_{2}\right)}{5 f^{\prime}}
\end{gathered}
$$

For $m=3$

$$
\begin{aligned}
& \mathbf{u} \approx \mathbf{U}_{3}=\mathbf{u}_{0}+\mathbf{u}_{1}+\mathbf{u}_{2}+\mathbf{u}_{3}=\mathbf{c}+\mathcal{N}\left(\mathbf{u}_{0}+\mathbf{u}_{1}+\mathbf{u}_{2}\right) \\
& =\beta-\frac{f(\beta)}{f^{\prime}(\beta)}-\frac{18 f\left(u_{0}+u_{1}\right)}{5 f^{\prime} \quad \frac{u_{0}+u_{1}+\beta}{2}-\frac{u_{0}+u_{1}-\beta}{2} \sqrt{\frac{3}{5}}+8 f^{\prime} \quad \frac{u_{0}+u_{1}+\beta}{2}+5 f^{\prime} \quad \frac{u_{0}+u_{1}+\beta}{2}+\frac{u_{0}+u_{1}-\beta}{2} \sqrt{\frac{3}{5}}} \\
& -\frac{18 f\left(u_{0}+u_{1}+u_{2}\right)}{5 f^{\prime} \frac{u_{0}+u_{1}+u_{2}+\beta}{2}-\frac{u_{0}+u_{1}+u_{2}-\beta}{2} \sqrt{\frac{3}{5}}+8 f^{\prime} \quad \frac{u_{0}+u_{1}+u_{2}+\beta}{2}+5 f^{\prime} \quad \frac{u_{0}+u_{1}+u_{2}+\beta}{2}+\frac{u_{0}+u_{1}+u_{2}-\beta}{2} \sqrt{\frac{3}{5}}},
\end{aligned}
$$

This formulation allows us to suggest the following Algorithm:

Algorithm 2(c): For a given $u_{0}$, approximate solution $u_{n+1}$ is computed by the following iterative scheme:

$$
\begin{aligned}
& y_{n}=u_{n}-\frac{f\left(u_{n}\right)}{f^{\prime}\left(u_{n}\right)}, \\
& z_{n}=y_{n}-\frac{18 f\left(y_{n}\right)}{5 f^{\prime} \frac{y_{n}+u_{n}}{2}-\frac{y_{n}-u_{n}}{2} \sqrt{\frac{3}{5}}+8 f^{\prime} \frac{y_{n}+u_{n}}{2}+5 f^{\prime} \frac{y_{n}+u_{n}}{2}+\frac{y_{n}-u_{n}}{2} \sqrt{\frac{3}{5}}}, \\
& u_{n+1}=z_{n}-\frac{\mathrm{n}=0,1,2, \ldots}{5 f^{\prime} \frac{z_{n}+u_{n}}{2}-\frac{z_{n}-u_{n}}{2} \sqrt{\frac{3}{5}}+8 f^{\prime} \frac{z_{n}+u_{n}}{2}+5 f^{\prime} \frac{z_{n}+u_{n}}{2}+\frac{z_{n}-u_{n}}{2} \sqrt{\frac{3}{5}}},
\end{aligned}
$$

For $m=4$, we have

$$
\begin{aligned}
& \mathbf{u} \approx \mathbf{U}_{4}=\mathbf{u}_{0}+\mathbf{u}_{1}+\mathbf{u}_{2}+\mathbf{u}_{3}+\mathbf{u}_{4}=\mathbf{c}+\mathcal{N}\left(\mathbf{u}_{0}+\mathbf{u}_{1}+\mathbf{u}_{2}+\mathbf{u}_{3}\right) \\
& =\beta-\frac{f(\beta)}{f^{\prime}(\beta)}-\frac{18 f\left(u_{0}+u_{1}\right)}{5 f^{\prime} \quad \frac{u_{0}+u_{1}+\beta}{2}-\frac{u_{0}+u_{1}-\beta}{2} \sqrt{\frac{3}{5}}+8 f^{\prime} \quad \frac{u_{0}+u_{1}+\beta}{2}+5 f^{\prime} \quad \frac{u_{0}+u_{1}+\beta}{2}+\frac{u_{0}+u_{1}-\beta}{2} \sqrt{\frac{3}{5}}} \\
& -\frac{18 f\left(u_{0}+u_{1}+u_{2}\right)}{5 f^{\prime} \quad \frac{u_{0}+u_{1}+u_{2}+\beta}{2}-\frac{u_{0}+u_{1}+u_{2}-\beta}{2} \sqrt{\frac{3}{5}}+8 f^{\prime} \quad \frac{u_{0}+u_{1}+u_{2}+\beta}{2}+5 f^{\prime} \quad \frac{u_{0}+u_{1}+u_{2}+\beta}{2}+\frac{u_{0}+u_{1}+u_{2}-\beta}{2} \sqrt{\frac{3}{5}}} \\
& -\frac{18 f(\tilde{u})}{5 f^{\prime} \frac{\tilde{u}+\beta}{2}-\frac{\tilde{u}-\beta}{2} \sqrt{\frac{3}{5}}+8 f^{\prime} \frac{\tilde{u}+\beta}{2}+5 f^{\prime} \frac{\tilde{u}+\beta}{2}+\frac{\tilde{u}-\beta}{2} \sqrt{\frac{3}{5}}} \text {. } \\
& \text { where } \quad \tilde{u}=u_{0}+u_{1}+u_{2}+u_{3}
\end{aligned}
$$

This formulation allows us to suggest the following four step algorithm. 
Algorithm 2(d): For a given $u_{0}$, approximate solution $u_{n+1}$ is computed by the following iterative scheme:

$y_{n}=u_{n}-\frac{f\left(u_{n}\right)}{f^{\prime}\left(u_{n}\right)}$,
$z_{n}=y_{n}-\frac{18 f\left(y_{n}\right)}{5 f^{\prime} \frac{y_{n}+u_{n}}{2}-\frac{y_{n}-u_{n}}{2} \sqrt{\frac{3}{5}}+8 f^{\prime} \frac{y_{n}+u_{n}}{2}+5 f^{\prime} \frac{y_{n}+u_{n}}{2}+\frac{y_{n}-u_{n}}{2} \sqrt{\frac{3}{5}}}$
$w_{n}=z_{n}-\frac{18 f\left(z_{n}\right)}{5 f^{\prime} \frac{z_{n}+u_{n}}{2}-\frac{z_{n}-u_{n}}{2} \sqrt{\frac{3}{5}}+8 f^{\prime} \frac{z_{n}+u_{n}}{2}+5 f^{\prime} \frac{z_{n}+u_{n}}{2}+\frac{z_{n}-u_{n}}{2} \sqrt{\frac{3}{5}}}$
$u_{n+1}=w_{n}-\frac{18 f\left(w_{n}\right)}{5 f^{\prime} \frac{w_{n}+u_{n}}{2}-\frac{w_{n}-u_{n}}{2} \sqrt{\frac{3}{5}}+8 f^{\prime} \frac{w_{n}+u_{n}}{2}+5 f^{\prime} \frac{w_{n}+u_{n}}{2}+\frac{w_{n}-u_{n}}{2} \sqrt{\frac{3}{5}}}, \quad \mathrm{n}=0,1,2, \ldots$

(2. 33)

\section{Convergence AnAlysis of Proposed Algorithms}

In this section, we determine the convergence order of proposed Algorithm 2(b), Algorithm 2(c) and Algorithm 2(d).

Theorem 3.1. For an open interval $I \subset R$. Let $f: I \rightarrow R$ and $\alpha \in I$ root of $f(u)=0$. Iff is differentiable and $u_{0}$ is sufficiently close to $\alpha$ then two step method defined by Algorithm 2(b) has third order of convergence and satisfies the error equation:

$$
e_{n+1}=c_{2}^{2} e_{n}^{3}-3 c_{2}^{3}-3 c_{2} c_{3} e_{n}^{4}+O\left(e_{n}^{5}\right) .
$$

Proof. Let $\alpha$ be simple zero of the function $f$ then expanding $f\left(u_{n}\right)$ and $f^{\prime}\left(u_{n}\right)$ about $\alpha$

$$
\begin{aligned}
& f\left(u_{n}\right)=f^{\prime}(\alpha)\left\{e_{n}+c_{2} e_{n}^{2}+c_{3} e_{n}^{3}+c_{4} e_{n}^{4}+c_{5} e_{n}^{5}+c_{6} e_{n}^{6}\right\} \\
& f^{\prime}\left(u_{n}\right)=f^{\prime}(\alpha)\left\{1+2 c_{2} e_{n}+3 c_{3} e_{n}^{2}+4 c_{4} e_{n}^{3}+5 c_{5} e_{n}^{4}+6 c_{6} e_{n}^{5}+7 c_{7} e_{n}^{6}\right\}
\end{aligned}
$$

where $c_{k}=\frac{1}{k !} \frac{f^{k}(\alpha)}{f^{\prime}(\alpha)}$ and $e_{n}=u_{n}-\alpha \quad \mathrm{k}=2,3, \ldots$

By using (3. 34 ), (3. 35 ) into (2. 24 ), we have

$$
\begin{gathered}
y_{n}=\alpha+c_{2} e_{n}^{2}+\left(2 c_{3}-2 c_{2}^{2}\right) e_{n}^{3}-\left(7 c_{2} c_{3}-3 c_{4}-4 c_{2}^{3}\right) e_{n}^{4} \\
+\left(4 c_{5}-10 c_{2} c_{4}-6 c_{3}^{2}+20 c_{3} c_{2}^{2}+8 c_{2}^{4}\right) e_{n}^{5}+O\left(e_{n}^{6}\right), \\
\begin{aligned}
f\left(y_{n}\right)=f^{\prime}(\alpha)\left\{c_{2} e_{n}^{2}+\left(2 c_{3}-2 c_{2}^{2}\right) e_{n}^{3}-\left(7 c_{2} c_{3}-3 c_{4}-4 c_{2}^{3}\right) e_{n}^{4}\right. \\
+\left(24 c_{3} c_{2}^{2}-12 c_{2}^{4}-6 c_{3}^{2}-10 c_{2} c_{4}+4 c_{5}\right\} e_{n}^{5}+O\left(e_{n}^{6}\right) .
\end{aligned}
\end{gathered}
$$

Expanding $f^{\prime}\left(\frac{u_{n}+y_{n}}{2}\right) f^{\prime}\left(\frac{y_{n}+u_{n}}{2}-\frac{y_{n}-u_{n}}{2} \sqrt{\frac{3}{5}}\right), f^{\prime}\left(\frac{y_{n}+u_{n}}{2}+\frac{y_{n}-u_{n}}{2} \sqrt{\frac{3}{5}}\right)$ by Taylor's series about $\alpha$

$$
\begin{aligned}
f^{\prime}\left(\frac{u_{n}+y_{n}}{2}\right)= & f^{\prime}(\alpha)\left\{1+c_{2} e_{n}+\left(c_{2}^{2}+\frac{3 c_{3}}{4}\right)+\left(\frac{7 c_{2} c_{3}}{2}-2 c_{2}^{3}+\frac{1}{2} c_{4}\right) e_{n}^{3}\right. \\
& \left.+\left(4 c_{2}^{4}+\frac{9 c_{2} c_{4}}{2}-\frac{37 c_{3} c_{2}^{2}}{4}+\frac{15 c_{3}^{2} c_{5}}{16}\right) e_{n}^{4}\right\}+O\left(e_{n}^{5}\right)
\end{aligned}
$$




$$
\begin{aligned}
& 8 f^{\prime}\left(\frac{u_{n}+y_{n}}{2}\right)=f^{\prime}(\alpha)\left\{8+8 c_{2} e_{n}+\left(8 c_{2}^{2}+6 c_{3}\right) e_{n}^{2}+\left(28 c_{2} c_{3}-16 c_{2}^{3}+4 c_{4}\right) e_{n}^{3}\right. \\
& +\left(\frac{5}{2} c_{5}+36 c_{2} c_{4}-74 c_{3} c_{2}^{2}+32 c_{2}^{4}+24 c_{3}^{2}\right) e_{n}^{4}+O\left(e_{n}^{5}\right) \\
& f^{\prime}\left(\frac{y_{n}+u_{n}}{2}-\frac{y_{n}-u_{n}}{2} \sqrt{\frac{3}{5}}\right)=f^{\prime}(\alpha)\left\{1+\left(5 c_{2}+\frac{1}{5} c_{2} \sqrt{15}\right) e_{n}+\left(c_{2}^{2}-\frac{1}{5} \sqrt{15} c_{2}^{2}+\frac{6}{5} c_{3}\right.\right. \\
& \left.+\frac{3}{10} \sqrt{15} c_{3}\right) e_{n}^{2}+\left(\frac{13}{5} c_{2} c_{3}-2 c_{2}^{3}+\frac{2}{5} \sqrt{15} c_{2}^{3}+\frac{7}{5} c_{4}\right. \\
& \left.-\frac{2}{5} \sqrt{15} c_{2} c_{3}+\frac{9}{25} \sqrt{15} c_{4}\right) e_{n}^{3}+\left(\frac{6}{5} c_{3}^{2}+\frac{11}{10} \sqrt{15} c_{3} c_{2}^{2}\right. \\
& -\frac{12}{25} \sqrt{15} c_{2} c_{4}+\frac{18}{5} c_{2} c_{4}+\frac{4}{5} \sqrt{15} c_{2}^{4}-7 c_{3} c_{2}^{2}+4 c_{4} \\
& \left.\left.+\frac{31}{20} c_{5}+\frac{2}{5} \sqrt{15} c_{5}\right) e_{n}^{4}+O\left(e_{n}^{5}\right)\right\} \\
& 5 f^{\prime}\left(\frac{y_{n}+u_{n}}{2}-\frac{y_{n}-u_{n}}{2} \sqrt{\frac{3}{5}}\right)=f^{\prime}(\alpha)\left\{5+\left(5 c_{2}+c_{2} \sqrt{15}\right) e_{n}+\left(5 c_{2}^{2}-\sqrt{15} c_{2}^{2}+6 c_{3}\right.\right. \\
& \left.+\frac{3}{2} \sqrt{15} c_{3}\right) e_{n}^{2}+\left(13 c_{2} c_{3}-10 c_{2}^{3}+2 \sqrt{15} c_{2}^{3}+7 c_{4}\right. \\
& \left.-2 \sqrt{15} c_{2} c_{3}+\frac{9}{5} \sqrt{15} c_{4}\right) e_{n}^{3}+\left(6 c_{3}^{2}+\frac{11}{2} \sqrt{15} c_{3} c_{2}^{2}\right. \\
& +18 c_{2} c_{4}-\frac{12}{5} \sqrt{15} c_{2} c_{4}-4 \sqrt{15} c_{2}^{4}-35 c_{3} c_{2}^{2}+20 c_{4} \\
& \left.\left.+\frac{31}{4} c_{5}+2 \sqrt{15} c_{5}\right) e_{n}^{4}+O\left(e_{n}^{5}\right)\right\} \\
& 5 f^{\prime}\left(\frac{y_{n}+u_{n}}{2}+\frac{y_{n}-u_{n}}{2} \sqrt{\frac{3}{5}}\right)=f^{\prime}(\alpha)\left\{5+\left(5 c_{2}-c_{2} \sqrt{15}\right) e_{n}+\left(5 c_{2}^{2}+\sqrt{15} c_{2}^{2}+6 c_{3}\right.\right. \\
& \left.-\frac{3}{2} \sqrt{15} c_{3}\right) e_{n}^{2}+\left(13 c_{2} c_{3}-10 c_{2}^{3}-2 \sqrt{15} c_{2}^{3}-\frac{9}{5} \sqrt{15} c_{4}\right. \\
& \left.+7 c_{4}+2 \sqrt{15} c_{2} c_{3}\right) e_{n}^{3}+\left(6 c_{3}^{2}-\frac{11}{2} \sqrt{15} c_{3} c_{2}^{2}+18 c_{2} c_{4}\right. \\
& +\frac{12}{5} \sqrt{15} c_{2} c_{4}+4 \sqrt{15} c_{2}^{4}-35 c_{3} c_{2}^{2}+20 c_{2}^{4}-2 \sqrt{15} c_{5} \\
& \left.\left.+\frac{31}{4} c_{5}\right) e_{n}^{4}+O\left(e_{n}^{5}\right)\right\} \text {. }
\end{aligned}
$$

From (3. 37 ), (3. 39$),(3.41),(3.42)$, we get

$$
\begin{aligned}
& \frac{f\left(y_{n}\right)}{5 f^{\prime}\left(\frac{y_{n}+u_{n}}{2}-\frac{y_{n}-u_{n}}{2} \sqrt{\frac{3}{5}}\right)+8 f^{\prime}\left(\frac{y_{n}+v_{n}}{2}\right)+f^{\prime}\left(\frac{y_{n}+u_{n}}{2}-\frac{y_{n}-u_{n}}{2} \sqrt{\frac{3}{5}}\right)} \\
&=c_{2} e_{n}^{2}+\left(2 c_{3}-3 c_{2}^{2}\right) e_{n}^{3}+O\left(e_{n}^{4}\right) .
\end{aligned}
$$


Substituting values from (3. 36 ), (3. 43 ) into (2. 25 ) and simplifying we get the error term of the Algorithm 2(b)

$$
\begin{aligned}
& u_{n+1}=\alpha+c_{2}^{2} e_{n}^{3}-\left(3 c_{2}^{3}-3 c_{2} c_{3}\right) e_{n}^{4}+O\left(e_{n}^{5}\right), \\
& e_{n+1}=c_{2}^{2} e_{n}^{3}-\left(3 c_{2}^{3}-3 c_{2} c_{3}\right) e_{n}^{4}+O\left(e_{n}^{5}\right) .
\end{aligned}
$$

Theorem 3.2. For an open interval $I \subset R$.Let $f: I \rightarrow R$ and $\alpha \in I$ be root of $f(u)=0$. If $f$ is differentiable and $u_{0}$ is sufficiently close to $\alpha$ then three step method defined by Algorithm 2(c) has fourth order of convergence and it satisfies the error equation:

$$
e_{n+1}=c_{2}^{3} e_{n}^{4}+\left(4 c_{3} c_{2}^{2}-4 c_{2}^{4}\right) e_{n}^{5}+O\left(e_{n}^{6}\right) .
$$

Proof. Considering again the equation (3. 44 ), we have

$$
z_{n}=\alpha+c_{2}^{2} e_{n}^{3}+\left(-3 c_{2}^{3}+3 c_{2} c_{3}\right) e_{n}^{4}+\left(-12 c_{3} c_{2}^{2}+6 c_{2}^{4}+4 c_{2} c_{4}+2 c_{3}^{2}\right) e_{n}^{5}+O\left(e_{n}^{6}\right),
$$

$f\left(z_{n}\right)=f^{\prime}(\alpha)\left\{c_{2}^{2} e_{n}^{3}+\left(3 c_{2} c_{3}-3 c_{2}^{3}\right) e_{n}^{4}+\left(-12 c_{3} c_{2}^{2}+2 c_{3}^{2}+6 c_{2}^{4}+4 c_{2} c_{4}\right) e_{n}^{5}+O\left(e_{n}^{6}\right)\right\}$.

Expanding $f^{\prime}\left(\frac{u_{n}+z_{n}}{2}\right), f^{\prime}\left(\frac{z_{n}+u_{n}}{2}-\frac{z_{n}-u_{n}}{2} \sqrt{\frac{3}{5}}\right), f^{\prime}\left(\frac{z_{n}+u_{n}}{2}+\frac{z_{n}-u_{n}}{2} \sqrt{\frac{3}{5}}\right)$ by Taylor series about $\alpha$

$$
\begin{aligned}
& 8 f^{\prime}\left(\frac{u_{n}+z_{n}}{2}\right)= f^{\prime}(\alpha)\left\{8+8 c_{2} e_{n}+6 c_{3} e_{n}^{2}+\left(8 c_{2}^{3}+4 c_{4}\right) e_{n}^{3}+\left(-24 c_{2}^{4}+36 c_{3} c_{2}^{2}\right.\right. \\
&+\left.\left.\frac{5}{2} c_{5}\right) e_{n}^{4}-\left(132 c_{2} c_{2}^{3}-52 c_{2} c_{3}^{2}-\frac{3}{2} c_{6}-44 c_{4} c_{2}^{2}-48 c_{2}^{5}\right) e_{n}^{5}+O\left(e_{n}^{5}\right)\right\}, \\
& \begin{aligned}
5 f^{\prime}\left(\frac{z_{n}+u_{n}}{2}-\frac{z_{n}-u_{n}}{2} \sqrt{\frac{3}{5}}\right)= & f^{\prime}(\alpha)\left\{5+\left(5 c_{2}+c_{2} \sqrt{15}\right) e_{n}+\left(6 c_{3}+\frac{3}{2} \sqrt{15} c_{3}\right) e_{n}^{2}\right. \\
& +\left(5 c_{2}^{3}-\sqrt{15} c_{2}^{3}+7 c_{4}+\frac{9}{5} \sqrt{15} c_{4}\right) e_{n}^{3}-\left(15 c_{2}^{4}-18 c_{3} c_{2}^{2}\right. \\
& \left.\left.-3 \sqrt{15} c_{2}^{4}+3 \sqrt{15} c_{3} c_{2}^{2}-\frac{31}{4} c_{5}-2 \sqrt{15} c_{5}\right) e_{n}^{4}+O\left(e_{n}^{5}\right)\right\},
\end{aligned}
\end{aligned}
$$

$$
\begin{aligned}
5 f^{\prime}\left(\frac{z_{n}+u_{n}}{2}+\frac{z_{n}-u_{n}}{2} \sqrt{\frac{3}{5}}\right) & =f^{\prime}(\alpha)\left\{5+\left(5 c_{2}-c_{2} \sqrt{15}\right) e_{n}+\left(6 c_{3}-\frac{3}{2} \sqrt{15} c_{3}\right) e_{n}^{2}\right. \\
& +\left(5 c_{2}^{3}+\sqrt{15} c_{2}^{3}+7 c_{4}-\frac{9}{5} \sqrt{15} c_{4}\right) e_{n}^{3}-\left(15 c_{2}^{4}-18 c_{3} c_{2}^{2}\right. \\
& \left.\left.+3 \sqrt{15} c_{2}^{4}-3 \sqrt{15} c_{3} c_{2}^{2}-\frac{31}{4} c_{5}+2 \sqrt{15} c_{5}\right) e_{n}^{4}+O\left(e_{n}^{5}\right)\right\} .
\end{aligned}
$$


From (3. 48$),(3.49)(3.50),(3.51)$, we have

$$
\begin{aligned}
& \frac{f\left(z_{n}\right)}{5 f^{\prime}\left(\frac{z_{n}+u_{n}}{2}-\frac{z_{n}-u_{n}}{2} \sqrt{\frac{3}{5}}\right)+8 f^{\prime}\left(\frac{u_{n}+y_{n}}{2}\right)+5 f^{\prime}\left(\frac{z_{n}+u_{n}}{2}+\frac{z_{n}-u_{n}}{2} \sqrt{\frac{3}{5}}\right)} \\
& =c_{2}^{2} e_{n}^{3}-\left(4 c_{2}^{3}-3 c_{2} c_{3}\right) e_{n}^{4}-\left(16 c_{3} c_{2}^{2}-10 c_{2}^{4}-4 c_{2} c_{4}-2 c_{3}^{2}\right) e_{n}^{5}+O\left(e_{n}^{6}\right) .
\end{aligned}
$$

Substituting values from (3. 47 ), (3. 52 ) into (2. 29 ) and simplifying we get the error term of the Algorithm 2(c)

$$
\begin{array}{r}
u_{n+1}=\alpha+c_{2}^{3} e_{n}^{4}+\left(4 c_{3} c_{2}^{2}-4 c_{2}^{4}\right) e_{n}^{5}+O\left(e_{n}^{6}\right), \\
e_{n+1}=c_{2}^{3} e_{n}^{4}+\left(4 c_{3} c_{2}^{2}-4 c_{2}^{4}\right) e_{n}^{5}+O\left(e_{n}^{6}\right) .
\end{array}
$$

Theorem 3.3. For an open interval $I \subset R$. Let $f: I \rightarrow R$ and $\alpha \in I$ be root of $f(u)=0$. If fis differentiable and $u_{0}$ is sufficiently close to $\alpha$ then four step method defined by Algorithm $2(d)$ has fifth order of convergence and it satisfies the error equation:

$$
e_{n+1}=c_{2}^{4} e_{n}^{5}+\left(5 c_{3} c_{2}^{3}-5 c_{2}^{5}\right) e_{n}^{6}+O\left(e_{n}^{7}\right) .
$$

Proof. Consider the equation (3.53)

$$
w_{n}=\alpha+c_{2}^{3} e_{n}^{4}+\left(4 c_{3} c_{2}^{2}-4 c_{2}^{4}\right) e_{n}^{5}+\left(5 c_{2}^{2} c_{4}+5 c_{2} c_{3}^{2}-20 c_{3} c_{2}^{2}+10 c_{2}^{5}\right) e_{n}^{6}+O\left(e_{n}^{7}\right) .
$$

Expanding $f\left(w_{n}\right)$ by Taylor's series about $\alpha$

$$
f\left(w_{n}\right)=f^{\prime}(\alpha)\left\{c_{2}^{3} e_{n}^{4}+\left(4 c_{3} c_{2}^{2}-4 c_{2}^{4}\right) e_{n}^{5}+\left(5 c_{4} c_{2}^{2}+5 c_{2} c_{3}^{2}-20 c_{3} c_{2}^{3}+10 c_{2}^{5}\right) e_{n}^{6}+O\left(e_{n}^{7}\right) .\right.
$$

Expanding $f^{\prime}\left(\frac{w_{n}+u_{n}}{2}\right), f^{\prime}\left(\frac{w_{n}+u_{n}}{2}-\frac{w_{n}-u_{n}}{2} \sqrt{\frac{3}{5}}\right), f^{\prime}\left(\frac{w_{n}+u_{n}}{2}+\frac{w_{n}-u_{n}}{2} \sqrt{\frac{3}{5}}\right)$ in terms of Taylor's series about $\alpha$

$$
\begin{aligned}
8 f^{\prime}\left(\frac{w_{n}+u_{n}}{2}\right)=f^{\prime}(\alpha)\left\{8+8 c_{2} e_{n}\right. & +6 c_{3} e_{n}^{2}+4 c_{4} e_{n}^{3}+\left(8 c_{2}^{4}+\frac{5}{2} c_{5}\right) e_{n}^{4}+\left(\frac{3}{2} c_{6}+44 c_{3} c_{2}^{3}\right. \\
- & \left.32 c_{2}^{5}\right) e_{n}^{5}+O\left(e_{n}^{6}\right), \\
5 f^{\prime}\left(\frac{w_{n}+u_{n}}{2}-\frac{w_{n}-u_{n}}{2} \sqrt{\frac{3}{5}}\right)= & f^{\prime}(\alpha)\left\{5+\left(5 c_{2}+c_{2} \sqrt{15}\right) e_{n}+\left(6 c_{3}+\frac{3}{2} \sqrt{15} c_{3}\right) e_{n}^{2}\right. \\
& +\left(7 c_{4}+\frac{9}{5} \sqrt{15} c_{4}\right) e_{n}^{3}+\left(2 \sqrt{15} c_{5}-\sqrt{15} c_{2}^{4}+5 c_{2}^{4}\right. \\
& \left.+\frac{31}{4} c_{5}\right) e_{n}^{4}+\left(\frac{33}{4} c_{6}-4 \sqrt{15} c_{3} c_{2}^{3}+4 \sqrt{15} c_{2}^{5}+23 c_{3} c_{2}^{3}\right. \\
& \left.+\frac{213}{100} \sqrt{15} c_{6}-20 c_{2}^{5}\right) e_{n}^{5}+O\left(e_{n}^{6}\right),
\end{aligned}
$$




$$
\begin{aligned}
5 f^{\prime}\left(\frac{w_{n}+u_{n}}{2}+\frac{w_{n}-u_{n}}{2} \sqrt{\frac{3}{5}}\right)= & f^{\prime}(\alpha)\left\{5+\left(5 c_{2}-c_{2} \sqrt{15}\right) e_{n}+\left(6 c_{3}-\frac{3}{2} \sqrt{15} c_{3}\right) e_{n}^{2}\right. \\
& +\left(7 c_{4}-\frac{9}{5} \sqrt{15} c_{4}\right) e_{n}^{3}+\left(-2 \sqrt{15} c_{5}+\sqrt{15} c_{2}^{4}+5 c_{2}^{4}\right. \\
& \left.+\frac{31}{4} c_{5}\right) e_{n}^{4}+\left(-4 \sqrt{15} c_{3} c_{2}^{3}-4 \sqrt{15} c_{2}^{5}+23 c_{3} c_{2}^{3}\right. \\
& \left.-20 c_{2}^{5}+\frac{33}{4} c_{6}-\frac{213}{100} \sqrt{15} c_{6}\right) e_{n}^{5}+O\left(e_{n}^{6}\right) .
\end{aligned}
$$

From (3. 57 ), (3. 58$),(3.59),(3.60)$ we have

$$
\begin{gathered}
\frac{f\left(w_{n}\right)}{5 f^{\prime}\left(\frac{w_{n}+u_{n}}{2}-\frac{w_{n}-u_{n}}{2} \sqrt{\frac{3}{5}}\right)+8 f^{\prime}\left(\frac{w_{n}+u_{n}}{2}\right)+5 f^{\prime}\left(\frac{w_{n}+u_{n}}{2}+\frac{w_{n}-u_{n}}{2} \sqrt{\frac{3}{5}}\right)} \\
=c_{2}^{3} e_{n+}^{4}\left(4 c_{3} c_{2}^{2}-5 c_{2}^{4}\right) e_{n}^{5}+O\left(e_{n}^{6}\right) .
\end{gathered}
$$

Substituting values from (3. 56 ), (3. 61 ) into (2. 33 ) and simplifying we get the error term of the Algorithm 2(d)

$$
\begin{gathered}
u_{n+1}=\alpha+c_{2}^{4} e_{n}^{5}+\left(5 c_{3} c_{2}^{3}-5 c_{2}^{5}\right) e_{n}^{6}+O\left(e_{n}^{7}\right), \\
e_{n+1}=c_{2}^{4} e_{n}^{5}+\left(5 c_{3} c_{2}^{3}-5 c_{2}^{5}\right) e_{n}^{6}+O\left(e_{n}^{7}\right) .
\end{gathered}
$$

The error equations (3. 45 ), (3. 54 ), and (3. 63 ) show that the Algorithm 2(b), Algorithm 2(c) and Algorithm 2(d) are third fourth \& fifth order convergent.

This completes the proof.

\section{NUMERICAL EXAMPLES AND APPLICATIONS}

In this section, we consider some nonlinear models related to Mathematics and Engineering which include continuous stirred tank reactor problem, van der Waal's equation, renowned population growth model, and nonlinear model formed due to motion of a particle on an inclined plane. We also include some of the nonlinear equations used by Chun [7] to elaborate the efficacy and effectiveness of the proposed algorithms. We obtain estimated simple root rather than the exact depending on the exactness $\varepsilon=10^{-100}$ of the computer. For computational work, we implement codes in Maple software and Matlab for graphical analysis, and the following stopping criterion is taken into account for entire computations:

$$
(i) .\left|u_{n+1}-u_{n}\right|<\varepsilon \quad \text { and } \quad(i i) . \mid f\left(u_{n+1} \mid<\varepsilon .\right.
$$

We compare the Newton-Raphson method (NM), Chun method (CM) [7], Weerakoon and Fernando method (WF) [34], Noor et al. methods [26] \{Algorithm 2.5 (NR1), Algorithm 2.8 (NR2), Algorithm 2.10 (NR3)\} with the Algorithm 2(b) (AG1), Algorithm 2(c) (AG2), Algorithm 2(d)(AG3). As for the convergence criteria, it was required that the distance of two consecutive approximations $\left(\delta\right.$ ) for the zero was less than $10^{-100}$. We display the number of iterations (IT), the approximate zero $u_{n}$ and the functional value $f\left(u_{n}\right)$ in Tables 
(1-5). The computational order of convergence (COC) (see [8]) is computed to check the behaviour of the proposed methods for presented examples and given by:

$$
\mathrm{COC} \approx \frac{\ln \left|u_{n+1}-u_{n}\right| /\left|u_{n}-u_{n-1}\right|}{\ln \left|u_{n}-u_{n-1}\right| /\left|u_{n-1}-u_{n-2}\right|}
$$

Example 4.1 (see [5](Continuous stirred tank reactor (CSTR)). Here, we consider the nonlinear equation stirred tank reactor i.e.,

$$
u^{4}+11.50 u^{3}+47.49 u^{2}+83.06325 u+51.23266875=0 .
$$

We use $x_{o}=-1.40$ as an initial guess for the computer programs in this example. The comparison of numerical results for this example is given in Table 1. It is clear from the numerical results obtained from Table 1 that the effectiveness and presentation of the new methods are much better than those of the other similar order methods. Fig 1 also shows the efficacy of proposed schemes by making the comparison of the iterative methods concerning to different methods in terms of the number of iterations.

Table 1: Numerical results for Equation 4. 66 .

\begin{tabular}{|c|c|c|c|c|c|}
\hline Methods & IT & $u_{n}$ & $f\left(u_{n}\right)$ & $\delta=\left|x_{n}-x_{n-1}\right|$ & $C O C$ \\
\hline NM & 6 & 1.4500000000000000 & $3.258864 \mathrm{e}-69$ & $1.798055 \mathrm{e}-35$ & 2.00000 \\
WF & 4 & 1.4500000000000000 & $4.450403 \mathrm{e}-85$ & $2.779472 \mathrm{e}-29$ & 2.99999 \\
NR1 & 4 & 1.4500000000000000 & $1.034328 \mathrm{e}-76$ & $1.424909 \mathrm{e}-26$ & 2.99973 \\
NR2 & 4 & 1.4500000000000000 & $1.545514 \mathrm{e}-87$ & $4.421829 \mathrm{e}-30$ & 2.99992 \\
NR3 & 3 & 1.4500000000000000 & $8.507087 \mathrm{e}-58$ & $1.609392 \mathrm{e}-15$ & 3.93555 \\
CM & 3 & 1.4500000000000000 & $3.887894 \mathrm{e}-56$ & $3.957471 \mathrm{e}-15$ & 3.92523 \\
AG1 & 3 & 1.4500000000000000 & $2.513368 \mathrm{e}-29$ & $1.120288 \mathrm{e}-10$ & 2.99999 \\
AG2 & 3 & 1.4500000000000000 & $3.280405 \mathrm{e}-69$ & $3.189433 \mathrm{e}-18$ & 3.96872 \\
AG3 & 2 & 1.4500000000000000 & $3.605181 \mathrm{e}-27$ & $2.298321 \mathrm{e}-06$ & 4.97036 \\
\hline
\end{tabular}




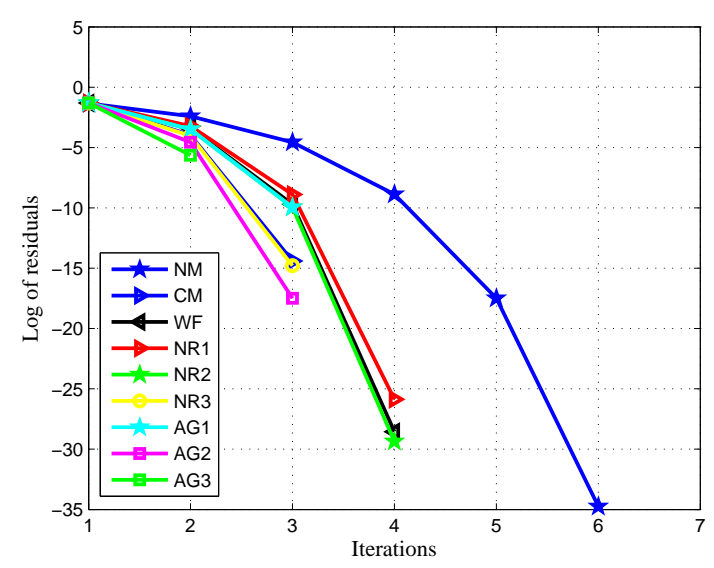

Figure 1: Log of residuals for Equation 4. 66 .

Example 4.2 (Van der Waal's equation see [33]). We consider the van der Waal's qquation representing the real and ideal behaviour of gass is prescribed as:

$$
\left(\mathcal{Q}+\frac{c p^{2}}{V^{2}}\right)(\mathcal{V}-p d)=p \mathcal{S} \mathcal{T},
$$

equation.(4. 67 ) can be transformed to the following nonlinear form:

$$
\mathcal{Q} \mathcal{V}^{3}-(p d \mathcal{Q}+p \mathcal{S T}) \mathcal{V}^{2}+c p^{2} \mathcal{V}-c p^{3} d
$$

after approperiatly choosing the needed parametres and unknown constants we can find the following nonlinear function:

$$
0.986 u^{3}-5.181 u^{2}+9.067 u-5.289=0 .
$$

Where the variable $\tilde{u}$ shows the volume of the gas. We take $\tilde{u}_{0}=3.10$ as an initial guess for computational evaluations. The mathematical computations for Eq.(4.69) are calculated in Table 2. Fig 2 shows the comparison of the iterative methods with respect to the number of iterations.

Table 2: Numerical results for Equation 4. 69.

\begin{tabular}{|c|c|c|c|c|c|}
\hline Methods & IT & $u_{n}$ & $f\left(u_{n}\right)$ & $\delta=\left|x_{n}-x_{n-1}\right|$ & $C O C$ \\
\hline NM & 12 & 1.9298462428478622 & $3.825655 \mathrm{e}-94$ & $2.693071 \mathrm{e}-47$ & 2.00000 \\
WF & 8 & 1.9298462428478622 & $1.000000 \mathrm{e}-126$ & $5.142285 \mathrm{e}-43$ & 3.00000 \\
NR1 & 8 & 1.9298462428478622 & $5.845408 \mathrm{e}-80$ & $2.086153 \mathrm{e}-27$ & 2.99975 \\
NR2 & 8 & 1.9298462428478622 & $0.000000 \mathrm{e}+00$ & $3.271773 \mathrm{e}-50$ & 3.00000 \\
NR3 & 7 & 1.9298462428478622 & $1.000000 \mathrm{e}-126$ & $8.979952 \mathrm{e}-39$ & 3.99905 \\
CM & 7 & 1.9298462428478622 & $0.000000 \mathrm{e}+00$ & $8.145024 \mathrm{e}-35$ & 3.99798 \\
AG1 & 7 & 1.9298462428478622 & $2.827856 \mathrm{e}-51$ & $9.577165 \mathrm{e}-18$ & 2.99612
\end{tabular}




\begin{tabular}{l|l|l|l|l|l|} 
AG2 & 6 & 1.9298462428478622 & $2.541997 \mathrm{e}-93$ & $3.372664 \mathrm{e}-24$ & 3.99999 \\
$\mathrm{AG} 3$ & 5 & 1.9298462428478622 & $1.807906 \mathrm{e}-71$ & $2.726914 \mathrm{e}-15$ & 4.96276 \\
\hline
\end{tabular}

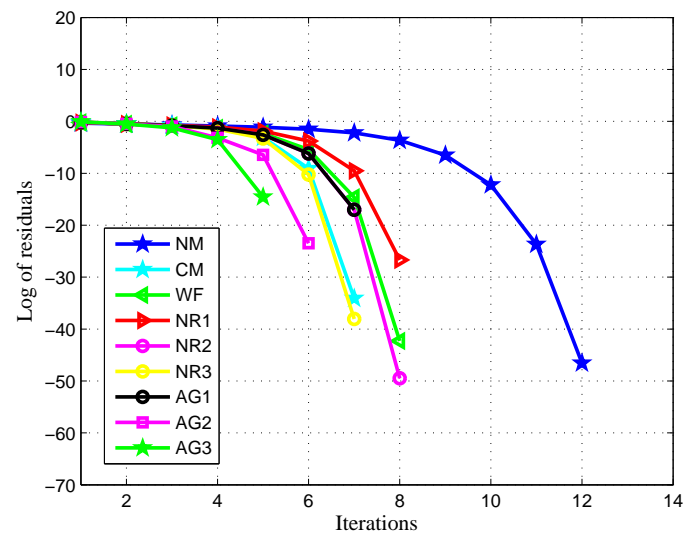

Figure 2: Log of residuals for Equation 4. 69 .

Table 2 and Figure 2 illustrate that the new methods, $A G 1, A G 2, A G 3$ require less number of iterations when compared with $N M, C M, W F, N R 1, N R 2, N R 3$ to meet the stopping criterium (4. 64 ). It is clear from the results of this example that the proposed methods converge more rapidly to the solution as compared to the already existed methods.

Example 4.3 (see [6](Population growth model)). Consider the nonlinear equation that appears in mathematical modeling of the growth of population over short periods of time:

$$
1564,000=1000,000 e^{\lambda}+435000\left(\frac{e^{\lambda}-1}{\lambda}\right)-1564000
$$

where $\lambda$ denotes the constant birth rate of population whose value needs to determined. For computational work, we take $x_{o}=1.5$ as an initial estimate. The numerical results for this problem are given in Table 3. Fig 3 show the comparison of the iterative methods with respect to number of iterations. 
Table 3: Numerical results for Equation 4. 70 .

\begin{tabular}{|c|c|c|c|c|c|}
\hline Methods & IT & $u_{n}$ & $f\left(u_{n}\right)$ & $\delta=\left|x_{n}-x_{n-1}\right|$ & $C O C$ \\
\hline NM & 7 & 0.1009979296857498 & $7.697779 \mathrm{e}-31$ & $1.104193 \mathrm{e}-18$ & 2.00000 \\
WF & 5 & 0.1009979296857498 & $3.049742 \mathrm{e}-54$ & $1.968666 \mathrm{e}-20$ & 2.99999 \\
CM & 5 & 0.1009979296857498 & $3.000000 \mathrm{e}-121$ & $7.646560 \mathrm{e}-45$ & 3.99979 \\
NR1 & 5 & 0.1009979296857498 & $5.620211 \mathrm{e}-45$ & $2.113407 \mathrm{e}-17$ & 2.99775 \\
NR2 & 4 & 0.1009979296857498 & $1.334042 \mathrm{e}-63$ & $1.648664 \mathrm{e}-23$ & 2.99979 \\
NR3 & 4 & 0.1009979296857498 & $3.875494 \mathrm{e}-41$ & $2.882365 \mathrm{e}-12$ & 3.92829 \\
AG1 & 4 & 0.1009979296857498 & $2.200930 \mathrm{e}-17$ & $4.197052 \mathrm{e}-08$ & 2.97866 \\
AG2 & 4 & 0.1009979296857498 & $3.380718 \mathrm{e}-67$ & $1.245760 \mathrm{e}-18$ & 3.98809 \\
AG3 & 3 & 0.1009979296857498 & $7.972330 \mathrm{e}-30$ & $1.644990 \mathrm{e}-07$ & 4.96899 \\
\hline
\end{tabular}

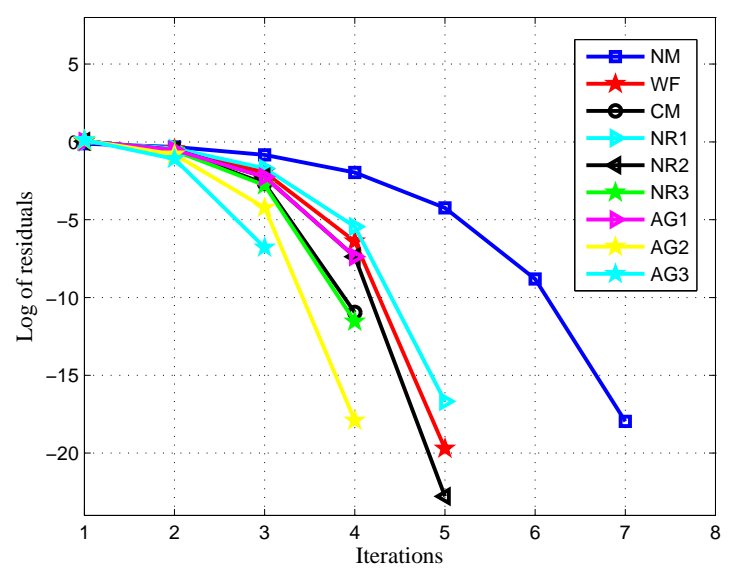

Figure 3: Log of residuals for Equation 4. 70 .

The second column of Table 3 demonstrates that new methods require less number of iteration to reach the stopping criteria (4.64) and the same for some cases when compared with different methods. It is clear from Table 3 and Fig 3 the approximate solutions obtained by the newly proposed methods can be compared with the well-known existing methods and performance is much better than the other methods. Computational order of convergence is also exhibited in the Table 3 which also verifies the convergence order of the methods.

Example 4.4 (see [26](Motion of particle on an Inclined plane)). 
We solve the nonlinear model formed due to the motion of a particle on an inclined plane whose angle of inclination, $\theta$ changes at a constant rate $\frac{d(\theta)}{d t}=w<0$ :

$$
x(t)=-\frac{g}{2 w^{2}}\left(\frac{e^{w t}-e^{-w t}}{2}-\sin w t\right) .
$$

We take $x_{o}=-0.36$ as an initial guess for computation evaluation. The comparison of numerical results for this problem are given in Table 4. Figure 4 represent the fall of residuals for this example.

Table 4: Numerical results for Equation 4. 71 .

\begin{tabular}{|c|c|c|c|c|c|}
\hline Methods & IT & $x_{n}$ & $f\left(u_{n}\right)$ & $\delta=\left|x_{n}-x_{n-1}\right|$ & $C O C$ \\
\hline NM & 6 & 0.3170617745729571 & $1.402507 \mathrm{e}-44$ & $2.575729 \mathrm{e}-22$ & 1.99999 \\
WF & 4 & 0.3170617745729571 & $7.807920 \mathrm{e}-54$ & $1.732945 \mathrm{e}-18$ & 2.99671 \\
NR1 & 4 & 0.3170617745729571 & $1.360230 \mathrm{e}-46$ & $3.708416 \mathrm{e}-16$ & 2.99170 \\
NR2 & 4 & 0.3170617745729571 & $1.292575 \mathrm{e}-55$ & $4.593526 \mathrm{e}-19$ & 2.99721 \\
NR3 & 3 & 0.3170617745729571 & $4.197484 \mathrm{e}-35$ & $1.056817 \mathrm{e}-09$ & 3.71019 \\
CM & 3 & 0.3170617745729571 & $7.409941 \mathrm{e}-34$ & $2.048702 \mathrm{e}-09$ & 3.99675 \\
AG1 & 4 & 0.3170617745729571 & $1.292567 \mathrm{e}-55$ & $4.593517 \mathrm{e}-19$ & 2.99720 \\
AG2 & 3 & 0.3170617745729571 & $1.445782 \mathrm{e}-44$ & $6.438628 \mathrm{e}-12$ & 3.99957 \\
AG3 & 3 & 0.3170617745729571 & $3.562374 \mathrm{e}-84$ & $9.233781 \mathrm{e}-18$ & 4.99999 \\
\hline
\end{tabular}

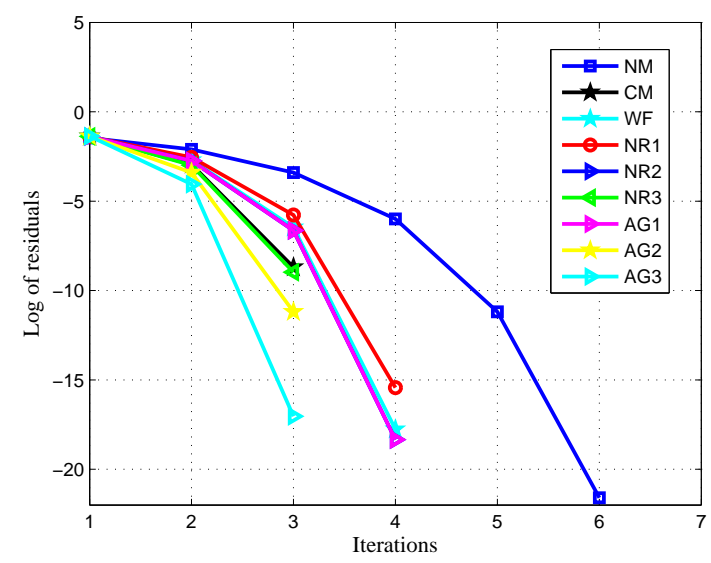

Figure 4: Log of residuals for Equation 4. 71 .

It is clear from computational results for the effectiveness and presentation of the new methods are much better and needs less number of iterations when compared with NM, NR1, NR2, NR3, CM, WF or same in cases (CM, NR3). In Table 4, and Fig 4 show the 
compatibility of numerical results for new iterative methods with the methods theoratical analysis.

Example 4.5 (Transcendental and Algebraic problems.).

To numerically analyze the suggested algorithms, we consider the following transcendental and algebraic equations used by Chun [7]:

$$
\begin{aligned}
f_{1}(u) & =(u-1)^{3}-1, & u_{o}=3.5, \\
f_{2}(u) & =\cos (u)-u, & u_{o}=1.7, \\
f_{3}(u) & =e^{u^{2}+7 u-30}-1, & u_{o}=3.5, \\
f_{4}(u) & =\sin ^{2}(u)-u^{2}+1, & u_{o}=-1, \\
f_{5}(u) & =u^{2}-e^{u}-3 u+2, & u_{o}=2 .
\end{aligned}
$$

In Table 5, we display the numerical results for examples $f_{1}(u), f_{2}(u), f_{3}(u), f_{4}(u), f_{5}(u)$ to validate the theoretical results.

Table 5: Numerical comparison between different algorithms for test problems $f_{1}(s)$ $f_{5}(s)$.

\begin{tabular}{|c|c|c|c|c|c|}
\hline Methods & IT & $u_{n}$ & $f\left(u_{n}\right)$ & $\delta=\left|u_{n}-u_{n-1}\right|$ & $C O C$ \\
\hline \multicolumn{7}{|c|}{$f_{1}(u)=(u-1)^{3}-1, u_{o}=3.5$} \\
\hline NM & 8 & 2.0000000000000000 & $2.056946 \mathrm{e}-42$ & $8.280390 \mathrm{e}-22$ & 2.00000 \\
CM & 6 & 2.0000000000000000 & $0.000000 \mathrm{e}+00$ & $2.827696 \mathrm{e}-94$ & 4.00000 \\
NF & 5 & 2.0000000000000000 & $9.839450 \mathrm{e}-37$ & $6.550899 \mathrm{e}-13$ & 2.98641 \\
NR2 & 7 & 2.0000000000000000 & $0.000000 \mathrm{e}+00$ & $1.240603 \mathrm{e}-83$ & 2.99999 \\
NR3 & 5 & 2.0000000000000000 & $4.040313 \mathrm{e}-120$ & $1.104328 \mathrm{e}-40$ & 3.00000 \\
AG1 & 5 & 2.0000000000000000 & $0.000000 \mathrm{e}+00$ & $5.864694 \mathrm{e}-26$ & 3.99224 \\
AG2 & 4 & 2.0000000000000000 & $3.312983 \mathrm{e}-40$ & $4.797695 \mathrm{e}-14$ & 2.99061 \\
AG3 & 3 & 2.00000000000000000 & $2.932850 \mathrm{e}-42$ & $3.144431 \mathrm{e}-11$ & 3.86663 \\
\hline \multicolumn{7}{|c|}{$f_{2}(u)=c 0 s(u)-u, \quad u_{0}=1.7$} \\
\hline NM & 7 & 0.7390851332151608 & $0.000000 \mathrm{e}+00$ & $3.254881 \mathrm{e}-65$ & 2.00000 \\
WF & 5 & 0.7390851332151606 & $0.000000 \mathrm{e}+00$ & $1.696999 \mathrm{e}-65$ & 3.00000 \\
NR1 & 5 & 0.7390851332151608 & $0.000000 \mathrm{e}+00$ & $5.225607 \mathrm{e}-73$ & 3.00000 \\
NR2 & 5 & 0.7390851332151606 & $0.000000 \mathrm{e}+00$ & $6.108403 \mathrm{e}-80$ & 3.00000 \\
NR3 & 4 & 0.7390851332151606 & $0.000000 \mathrm{e}+00$ & $2.241483 \mathrm{e}-55$ & 3.99987 \\
CM & 4 & 0.7390851332151606 & $0.000000 \mathrm{e}+00$ & $1.867468 \mathrm{e}-53$ & 3.99983 \\
AG1 & 4 & 0.7390851332151606 & $9.975704 \mathrm{e}-80$ & $1.069276 \mathrm{e}-26$ & 2.99942 \\
AG2 & 4 & 0.7390851332151620 & $0.000000 \mathrm{e}+00$ & $4.125693 \mathrm{e}-62$ & 3.99995 \\
AG3 & 2 & 0.7390851332151620 & $7.930209 \mathrm{e}-24$ & $7.243271 \mathrm{e}-05$ & 4.88893 \\
\hline \multicolumn{7}{|c|}{$f_{3}(u)=e^{u^{2}+7 u-30}-1}$, & $1.015348 \mathrm{e}-20$ & $8.052339 \mathrm{e}-05$ & 4.86465 \\
\hline NM & 13 & 3.0000000000000000 & $1.516930 \mathrm{e}-47$ & $4.212111 \mathrm{e}-25$ & 2.00000 \\
CM & 9 & 3.0000000000000000 & $0.000000 \mathrm{e}+00$ & $2.884803 \mathrm{e}-88$ & 4.00000 \\
WF & 9 & 3.0000000000000000 & $5.066487 \mathrm{e}-71$ & $4.069243 \mathrm{e}-25$ & 2.99979 \\
\end{tabular}




\begin{tabular}{|c|c|c|c|c|c|} 
NR1 & 10 & 3.0000000000000000 & $0.000000 \mathrm{e}+00$ & $3.108113 \mathrm{e}-46$ & 3.00000 \\
NR2 & 8 & 3.0000000000000000 & $7.833171 \mathrm{e}-38$ & $5.183816 \mathrm{e}-14$ & 2.99434 \\
NR3 & 9 & 3.0000000000000002 & $0000000 \mathrm{e}+00$ & $2.495598 \mathrm{e}-99$ & 3.99999 \\
AG1 & 8 & 3.0000000000000510 & $7.473796 \mathrm{e}-38$ & $5.103296 \mathrm{e}-14$ & 2.99999 \\
AG2 & 7 & 3.0000000000000000 & $7.004102 \mathrm{e}-93$ & $1.173100 \mathrm{e}-24$ & 3.99523 \\
AG3 & 6 & 3.0000000000000000 & $1.159203 \mathrm{e}-87$ & $5.440327 \mathrm{e}-19$ & 4.95234 \\
\hline \multicolumn{6}{|c|}{$f_{4}(u)=\sin ^{2}(u)-u^{2}+1$} \\
\hline NM & 7 & 1.4044916482153412 & $1.044527 \mathrm{e}-50$ & $7.327881 \mathrm{e}-26$ & 2.00000 \\
CM & 6 & 1.4044916482153412 & $2.10000 \mathrm{e}-127$ & $7.12655 \mathrm{e}-68$ & 3.99999 \\
WF & 5 & 1.4044916482153412 & $8.904296 \mathrm{e}-89$ & $3.791998 \mathrm{e}-30$ & 3.00010 \\
NR1 & 16 & 1.4044916482153412 & $1.095509 \mathrm{e}-20$ & $1.531728 \mathrm{e}-07$ & 2.97384 \\
NR2 & 5 & 1.4044916482153412 & $5.953595 \mathrm{e}-94$ & $7.309921 \mathrm{e}-32$ & 3.00005 \\
NR3 & 5 & 1.4044916482153412 & $1.000000 \mathrm{e}-127$ & $6.860606 \mathrm{e}-97$ & 3.99935 \\
AG1 & 4 & 1.4044916482153412 & $1.773310 \mathrm{e}-31$ & $4.881813 \mathrm{e}-11$ & 3.03474 \\
AG2 & 3 & 1.4044916482153412 & $7.210014 \mathrm{e}-25$ & $8.814637 \mathrm{e}-07$ & 4.33293 \\
AG3 & 3 & 1.4044916482153412 & $3.720234 \mathrm{e}-48$ & $3.310354 \mathrm{e}-10$ & 4.99921 \\
\hline \multicolumn{7}{|c|}{$f_{5}(u)=u^{2}-e^{u}-3 u+2}$, & $u_{0}=2$ \\
\hline NM & 7 & 0.2575302854398608 & $2.117415 \mathrm{e}-111$ & $7.743422 \mathrm{e}-56$ & 2.00000 \\
WF & 5 & 0.2575302854398608 & $5.935509 \mathrm{e}-103$ & $1.630507 \mathrm{e}-11$ & 3.00999 \\
NR1 & 4 & 0.2575302854398608 & $1.218871 \mathrm{e}-41$ & $5.694586 \mathrm{e}-14$ & 2.91743 \\
NR2 & 4 & 0.2575302854398608 & $3.376128 \mathrm{e}-53$ & $1.007610 \mathrm{e}-17$ & 2.97840 \\
CM & 4 & 0.2575302854398608 & $1.236660 \mathrm{e}-114$ & $9.463123 \mathrm{e}-29$ & 3.97784 \\
NR3 & 3 & 0.2575302854398608 & $9.720474 \mathrm{e}-29$ & $2.979348 \mathrm{e}-07$ & 4.65461 \\
AG1 & 3 & 0.2575302854398608 & $3.732105 \mathrm{e}-17$ & $1.041853 \mathrm{e}-05$ & 3.48762 \\
AG2 & 3 & 0.2575302854398607 & $3.162123 \mathrm{e}-45$ & $3.182065 \mathrm{e}-11$ & 4.14451 \\
AG3 & 2 & 0.2575302854398607 & $2.735843 \mathrm{e}-18$ & $1.569721 \mathrm{e}-03$ & 4.94443 \\
\hline
\end{tabular}

The second column in Table 5 represent the number of iterations for different nonlinear functions along with initial guess $u_{0}$. A comparative representation of the number of iterations is presented, needed for different methods with our developed methods using the stopping criteria (4. 64 ) \& accuracy $\varepsilon=10^{-100}$. It is clear from Table 5 that settling the same convergence criteria for all the methods, the number of iterations required for the new methods remains less or equal in some cases than the number of iterations needed by the other methods of the same order. The computational order of convergence is computed to validate theoratical results and it varies for all the methods.

\section{CONCLUSION}

In this manuscript, we have presented the new third, fourth-order, and fifth-order convergent iterative schemes by using the decomposition technique and quadrature formula. The efficiency and performance of these schemes are checked with the support of some numerical examples. A comparative analysis of these newly established schemes with some known schemes existing in the literature is also presented to validate the theoretical results. The computational numerical results and graphical analysis show the supermacy of the proposed schemes over the other iterative schemes; see Tables 1-5 and Figures 1-4. 


\section{CONFLICT OF INTEREST}

The authors agree with the contents of the manuscript, and there is no conflict of interest among the authors.

\section{AUTHOR'S CONTRIBUTIONS}

All authors contributed equally and significantly in writing this article. All authors read and approved the final manuscript.

\section{ACKNOWLEDGMENTS}

The author's would like to thank the Rector, COMSATS University Islamabad, Islamabad Pakistan, for providing excellent research and academic environments.

\section{REFERENCES}

[1] S. Abbasbandy, Improving Newton's Raphson method for nonlinear equations by modified adomian decomposition method, Appl. Math. Comput, 145 (2003) 887-893.

[2] A. R. Alharbi, M. I. Faisal, F. A. Shah, M. Waseem, R. Ullah, S. Sherbaz, Higher order numerical approaches for nonlinear equations by decomposition technique, IEEE Acess. (2020) 44329-44337.

[3] F. Ali, W. Aslam, A. Rafiq, New Family of iterative methods for solving nonlinear models, Discrete Dyn Nat Soc, 2018, 1-12. Article ID 9619680.

[4] F. Ali, W. Aslam, I. Khalid, A. Nadeem, Iteration methods with an auxiliary function for nonlinear equations, J. Math. 2020, 1-15. Article ID 7356408.

[5] R. Behl, S. Bhalla, E. Martínez, and M. A. Alsulami, Derivative-free King's scheme for multiple zeros of nonlinear functions, Mathematics, 9 (2021) 1-14.

[6] R. L. Burden, J. D. Faires, Numerical Analysis, PWS Publishing Company, Boston, 2001.

[7] C. Chun, Iterative methods improving Newton's method by the decomposition method, Comput. Math. Appl. 50 (2005) 1559-1568.

[8] A. Cordero, J. R. Torregrosa, Variants of Newton's method using fifth-order quadrature formulas, Appl. Math. Comput., 190 (2007) 686-698.

[9] V. Daftardar-Gejji, H. Jafari, An iterative method for solving nonlinear functional equations, J. Math. Anal. Appl. 316 , No. 2 (2006) 753-763.

[10] T. Eftekhari, Interval extension of the Halley method and its modified method for finding the root enclosures of nonlinear equations, Comput. Meth. Differ Equ. 8, No. 2 (2020) 222-235.

[11] T. Eftekhari, An efficient class of multipoint root-solvers with and without memory for nonlinear equations, Acta. Math. Vietnam. 41 (2016) 299-311.

[12] T. Eftekhari, Producing an interval extension of the King method, Appl. Math. Comput. 260 (2015) $288-291$.

[13] T. Eftekhari, A new proof of interval extension of the classic Ostrowski's method and its modified method for computing the enclosure solutions of nonlinear equations, Numer. Algorithms. 69 (2015), 157-165.

[14] T. Eftekhari, A new family of four-step fifteenth-order root-finding methods with high efficiency index, Comput. Meth. Diff Equ,3 (2015) 51-58.

[15] T. Eftekhari, On Some iterative methods with memory and high efficiency index for solving nonlinear equations, Int. J. of Diff. Equ. 2014 (2014) 1-6. Article ID 495357.

[16] T. Eftekhari, A New sixth-order Steffensen-type iterative method for solving nonlinear equations, Int. J. Anal. 2014 (2014) 1-5. Article ID 685796.

[17] M. Frontini, E. Sormani, Third-Order methods from quadrature formulae for solving systems of nonlinear equations, Appl. Math. Comput. 149 (2004) 771-782.

[18] J. H. He, A new iteration method for solving algebraic equations, Appl. Math. Comput,135 (2003), 81-84.

[19] S. Huang, A. Rafiq, M. R. Shahzad, New higher order iterative methods for solving nonlinear equations, Hacettepe J. Math. Stat. 46, No. 4 (2017) 77-91.

[20] A. Naseem, M. A. Rehmani, T. Abdeljawad, Y. Chu, Novel iteration schemes for computing zeros of nonlinear equations with engineering applications and their dynamics, IEEE Acess, 27 (2021) 92246-92262. 
[21] M. A. Noor, Variational inequalities and approximation, Punjab Univer. J. Math, 8 (1975), $30-57$.

[22] M. A. Noor, New approximation schemes for solving general variational inequalities, J. Math. Anal. Appl, 251 (2000), 217-229

[23] M. A. Noor, Some developments in general variational inequalities, Appl. Math. Comput. 152 (2004), 199277

[24] M. A. Noor, K. I. Noor and M. Th. Rassias, New Trends in general variational inequalities, Acta Appl. Mathematica, 170, No. 1 (2020), 981-1046

[25] M. A. Noor, K. I. Noor, E. Al-Said, Some new iterative methods for nonlinear equations, Math. Prob. Eng. 2010 (2010) 1-12.

[26] M. A. Noor, M. Waseem, K. I. Noor, M. A. Amir, New iterative technique for solving nonlinear equations, Appl. Math. Comput., 265 (2015), 1115-1129.

[27] M. A. Noor, Fifth order convergent iterative method for solving nonlinear equation using quadrature formula, J. Math. Cont. Sci. Appl. 4, No. 1 (2018) 95-104.

[28] O. Ogbereyivwe, K. Muka, Multistep quadrature based methods for nonlinear system of equations with singular Jacobian, J. Appl. Math. Phy. 7 (2019) 702-725.

[29] G. Sana, M. A. Noor, K. I. Noor, Some multistep iterative methods for nonlinear equation using quadrature rule, Int. J. Anal. App. 18, No. 6 (2020), 920-938.

[30] M. Saqib, M. Iqbal, Some multi-step iterative methods for solving nonlinear equations, Open. J. Math. Sci. 58, No. 8 (2017) 1589-1597.

[31] H. M. Srivastava, J. Iqbal, M. Arif, A. Khan, Y. S. Gasimov, R. Chinram 5, A new application of gauss quadrature Method for solving systems of nonlinear equations, Symmetry. (2021), 1-12.

[32] J. F. Traub, Iterative methods for the solution of equations, Chelsea publishing company, New York, 1977.

[33] V. D. Waals, J. Diderik, Over de continuiteit van den gasen vloeistoftoestand (on the continuity of the gas and liquid state), Ph.D. thesis, Leiden, The Netherlands, 1873.

[34] S. Weerakoon, T. G. I. Fernando, A variant of Newton's method with accelerated third-order convergence, Appl. Math. Letter. 13, No. 8 (2000) 87-93.

[35] X. Zhang, F. A. Shah, Y. Li, L. Yan, A. Q. Baig, M. R. Farahani, A family of fifth-order convergent methods for solving nonlinear equations using variational iteration technique, J. Inf. Optim. Sci. 39 (2018) 673-694. 